\title{
A note on the number of solutions of the generalized Ramanujan-Nagell equation $x^{2}-D=k^{n}$
}

\author{
by \\ MaOHuA LE (Zhanjiang)
}

1. Introduction. Let $\mathbb{Z}, \mathbb{N}$ be the sets of integers and positive integers respectively. Let $D$ be a nonzero integer, and let $k$ be a positive integer such that $k>1$ and $\operatorname{gcd}(D, k)=1$. Further let $N(D, k)$ denote the number of solutions $(x, n)$ of the generalized Ramanujan-Nagell equation

$$
x^{2}-D=k^{n}, \quad x, n \in \mathbb{N} .
$$

There have been many papers concerned with upper bounds for $N(D, k)$. Let $C_{i}(i=1,2, \ldots)$ denote effectively computable absolute constants. The known results include the following:

1 (Apéry $[1,2])$. If $D<0, k$ is a prime and $(D, k) \neq(-7,2)$, then $N(D, k) \leq 2$.

2 (Beukers [3]). If $D<-7$, then $N(-23,2)=N\left(-2^{r}+1,2\right)=2$ for some $r \in \mathbb{N}$, otherwise $N(D, 2) \leq 1$.

3 (Le [10]). If $D<0, k$ is an odd prime and $|D|>C_{1}$, then $N\left(-3 s^{2}-\right.$ $\left.1,4 s^{2}+1\right)=2$ for some $s \in \mathbb{N}$, otherwise $N(D, k) \leq 1$.

4 (Xu and Le [15]). If $D<0,2 \nmid k$ and $|D|>C_{2}$, then

$$
N(D, k) \leq \begin{cases}2^{\omega(k)-1}+1 & \text { if } D=-3 s^{2} \pm \text { and } k^{r}=4 s^{2} \mp 1 \\ 2^{\omega(k)-1} & \text { otherwise, }\end{cases}
$$

where $\omega(k)$ is the number of distinct prime factors of $k$.

5 (Beukers [3, 4]). If $D>0$ and $k$ is a prime, then $N(D, k) \leq 4$.

6 (Le [9]). If $D>0$, then $N\left(2^{2 r}-3 \cdot 2^{r+1}+1,2\right)=4$ for some $r \in \mathbb{N}$, otherwise $N(D, 2) \leq 3$.

7 (Le [8]). If $D>0, k$ is an odd prime and $\max (D, k)>C_{3}$, then $N(D, k) \leq 3$.

1991 Mathematics Subject Classification: 11D61.

Supported by the National Natural Science Foundation of China and the Guangdong Provincial Natural Science Foundation. 
8 (Chen and Le [6]). If $D>0,2 \nmid k$ and $\max (D, k)>C_{4}$, then $N(D, k) \leq$ $3 \cdot 2^{\omega(k)-1}+1$.

So far we have not been able to find references to the case where $2 \mid k$ and $k$ is not a power of 2 . In this note we prove the following general result: Then

TheOREM. Let $\omega(D)$ be the number of distinct prime factors of $|D|$.

$$
N(D, k) \leq \begin{cases}2^{\omega(D)+1} & \text { if } D<0 \\ 2^{\omega(D)+1}+1 & \text { if } D>0\end{cases}
$$

\section{Preliminaries}

LEMMA 1. If $D>0$ and $D$ is not a square, then (1) has at most one solution $(x, n)$ with $k^{n}<\sqrt{D}$.

Proof. By [7, Theorem $10 \cdot 8 \cdot 2]$, if $k^{n}<\sqrt{D}$, then $x / 1$ must be a convergent of $\sqrt{D}$ with $x / 1>\sqrt{D}$. Notice that $\sqrt{D}$ has at most one convergent $p / q$ satisfying $q=1$ and $p / q>\sqrt{D}$. The lemma is proved.

LEMMA 2. If $k$ is not a square and the equation

$$
X^{2}-k Y^{2}=D, \quad X, Y \in \mathbb{Z}, \operatorname{gcd}(X, Y)=1
$$

has solutions $(X, Y)$, then all solutions of $(2)$ can be put into at most $2^{\omega(D)-1}$ classes. Moreover, every solution $(X, Y)$ in the class $T$ can be expressed as

$$
X+Y \sqrt{k}=\left(X_{0}+\delta Y_{0} \sqrt{k}\right)(u+v \sqrt{k}), \quad \delta \in\{-1,1\},
$$

where $\left(X_{0}, Y_{0}\right)$ is a fixed positive integer solution in $T,(u, v)$ is a solution of the equation

$$
u^{2}-k v^{2}=1, \quad u, v \in \mathbb{Z} .
$$

Proof. This is a special case of [11, Theorem 2] for $D_{1}=1$ and $z=1$.

Lemma 3. For $1 \leq D \leq 5$, the equation

$$
X^{2}+D=Y^{n}, \quad X, Y, n \in \mathbb{N}, \operatorname{gcd}(X, Y)=1, n>3
$$

has no solutions $(X, Y, n)$.

Proof. This follows immediately from the results of [5], [12] and [13].

Lemma 4. For $r, r^{\prime} \in \mathbb{N}$ with $r<r^{\prime}$, let $S, S^{\prime}$ be the sets of positive integer solutions $(u, v)$ of (3) satisfying

$$
k^{r} \mid v, \quad \operatorname{gcd}\left(k, v / k^{r}\right)=1,
$$

and

$$
k^{r^{\prime}} \mid v, \quad \operatorname{gcd}\left(k, v / k^{r^{\prime}}\right)=1,
$$


respectively. If $S \neq \emptyset, S^{\prime} \neq \emptyset,(U, V)$ and $\left(U^{\prime}, V^{\prime}\right)$ are least solutions of $S$ and $S^{\prime}$ respectively, then

$$
U^{\prime}+V^{\prime} \sqrt{k}=(U+V \sqrt{k})^{k^{r^{\prime}-r}} .
$$

Proof. Since $(U, V)$ is the least solution of $S, U+\left(V / k^{r}\right) \sqrt{k^{2 r+1}}$ is the fundamental solution of the equation

$$
u^{\prime 2}-k^{2 r+1} v^{\prime 2}=1, \quad u^{\prime}, v^{\prime} \in \mathbb{Z} .
$$

Further, since $\left(U^{\prime}, V^{\prime} / k^{r}\right)$ is a positive integer solution of (7), there exists a suitable $t \in \mathbb{N}$ such that

$$
U^{\prime}+\frac{V^{\prime}}{k^{r}} \sqrt{k^{2 r+1}}=\left(U+\frac{V}{k^{r}} \sqrt{k^{2 r+1}}\right)^{t},
$$

whence we get

$$
U^{\prime}+V^{\prime} \sqrt{k}=(U+V \sqrt{k})^{t} .
$$

Let $s=[(t-1) / 2]$. From $(8)$, we get

$$
V^{\prime}=V \sum_{i=0}^{s}\left(\begin{array}{c}
t \\
2 i+1
\end{array}\right) U^{t-2 i-1}\left(k V^{2}\right)^{i} .
$$

Notice that $r<r^{\prime}, k^{r}\left|V, k^{r^{\prime}}\right| V^{\prime}$ and $\operatorname{gcd}\left(k, V / k^{r}\right)=\operatorname{gcd}(k, U)=1$. We see from (9) that $k \mid t$ and

$$
\frac{V^{\prime}}{V}=\sum_{i=0}^{s}\left(\begin{array}{c}
t \\
2 i+1
\end{array}\right) U^{t-2 i-1}\left(k V^{2}\right)^{i} \equiv 0\left(\bmod k^{r^{\prime}-r}\right) .
$$

Let $k=p_{1}^{\alpha_{1}} \ldots p_{m}^{\alpha_{m}}$ be the factorization of $k$, and let $p_{j}^{\beta_{j}} \| t$ for $j=$ $1, \ldots, m$. Further, let $p_{j}^{\gamma_{i j}} \| 2 i+1$ for any $i \in \mathbb{N}$ and $j=1, \ldots, m$. Then we have $\gamma_{i j} \leq(\log (2 i+1)) / \log p_{j}<2 i$, and hence,

$$
\begin{aligned}
\left(\begin{array}{c}
t \\
2 i+1
\end{array}\right) U^{t-2 i-1}\left(k V^{2}\right)^{i} & =t U^{t-2 i-1}\left(\begin{array}{c}
t-1 \\
2 i
\end{array}\right) \frac{\left(k V^{2}\right)^{i}}{2 i+1} \\
& \equiv 0\left(\bmod p_{j}^{\beta_{j}+1}\right), \quad j=1, \ldots, m .
\end{aligned}
$$

By (10) and (11), we get $k^{r^{\prime}-r} \mid t$ and $t=k^{r^{\prime}-r} t_{1}$, where $t_{1} \in \mathbb{N}$. Therefore, by (4), if $\left(U^{\prime}, V^{\prime}\right)$ satisfies (6), then it is the least positive integer solution of (3) satisfying (5). The lemma is proved.

Lemma 5 ([14, Theorem I.2]). If $k$ is not a square and $(x, n)$ is a solution of (1) satisfying $k^{n} \geq 4^{1+s / r} D^{2+s / r}$ for some $r, s \in \mathbb{N}$, then

$$
\left|\frac{x^{\prime}}{k^{n^{\prime} / 2}}-1\right|>\frac{8}{2187 k^{n(3+\nu / 2)}}\left(\frac{81 k^{n}}{4}\right)^{1 / s} k^{-n^{\prime}(1+\nu) / 2}
$$

for any $x^{\prime}, n^{\prime} \in \mathbb{N}$ with $2 \nmid n^{\prime}$, where $\nu$ satisfies $k^{n \nu}=9\left(81 k^{n} / 4\right)^{r / s}$. 
LEMMA 6. If $k$ is not a square and (1) has a solution $(x, n)$ such that $k^{n} \geq \max \left(10^{5}, 4^{3} D^{4}\right)$, then every solution $\left(x^{\prime}, n^{\prime}\right)$ of $(1)$ with $2 \nmid n^{\prime}$ satisfies $n^{\prime}<39 n$.

P r o of. Let $\left(x^{\prime}, n^{\prime}\right)$ be a solution of (1) with $2 \nmid n^{\prime}$. Then

$$
\left|\frac{x^{\prime}}{k^{n^{\prime} / 2}}-1\right|=\frac{D}{k^{n^{\prime} / 2}\left(k^{n^{\prime} / 2}+x^{\prime}\right)}<\frac{D}{k^{n^{\prime}}} .
$$

Since $k^{n} \geq \max \left(10^{5}, 4^{3} D^{4}\right)$, by Lemma 5 , we get

$$
\left|\frac{x^{\prime}}{k^{n^{\prime} / 2}}-1\right|>\frac{8}{2187 k^{n(3+\nu / 2)}}\left(\frac{81 k^{n}}{4}\right)^{1 / 2} k^{-n^{\prime}(1+\nu) / 2},
$$

where

$$
\nu=\frac{\log 9}{\log k^{n}}+\frac{\log (81 / 4)}{2 \log k^{n}}+\frac{1}{2}<0.8215 .
$$

The combination of (12) and (13) yields

$$
\frac{D}{k^{n^{\prime}}}>\frac{8}{2187 k^{n(3+\nu / 2)}}\left(\frac{81 k^{n}}{4}\right)^{1 / 2} k^{-n^{\prime}(1+\nu) / 2} .
$$

Since $D \leq\left(k^{n} / 64\right)^{1 / 4}$ and $k^{n} \geq 10^{5}$, from (5) we get

$$
k^{n(6+\nu) / 2}>60.75 D k^{n(5+\nu) / 2}>k^{n^{\prime}(1-\nu) / 2} .
$$

This implies that

$$
n^{\prime}<\left(\frac{6+\nu}{1-\nu}\right) n .
$$

Substituting (14) into (17), we obtain $n^{\prime}<39 n$. The lemma is proved.

3. Proof of Theorem. By the known results of [1]-[4], we may assume that $k$ is not a prime power.

If $k$ is a square, then from (1) we get $x+k^{n / 2}=D_{1}$ and $x-k^{n / 2}=D_{2}$, where $D_{1}, D_{2}$ are integers satisfying $D_{1} D_{2}=D, \operatorname{gcd}\left(D_{1}, D_{2}\right) \leq 2, D_{1}>0$ and $D_{1}>D_{2}$. Notice that there exist at most $2^{\omega(D)-1}$ such pairs $\left(D_{1}, D_{2}\right)$. So we have $N(D, k) \leq 2^{\omega(D)-1}$ in this case. From the above, we may assume that $k$ is not a square. Similarly, we see that (1) has at most $2^{\omega(D)-1}$ solutions $(x, n)$ with $2 \mid n$.

If $(x, n)$ is a solution of (1) with $2 \nmid n$, then the equation (2) has a solution $(X, Y)=\left(x, k^{(n-1) / 2}\right)$. By Lemma 2 , all solutions $(X, Y)$ of $(2)$ can be put into at most $2^{\omega(D)-1}$ classes.

First we consider the case $D>0$. We now suppose that (1) has five solutions $\left(x_{i}, n_{i}\right)(i=1, \ldots, 5)$ such that $n_{1}<\ldots<n_{5}, k^{n_{1}}<\sqrt{D}, 2 \nmid n_{i}(i=$ $1, \ldots, 5)$ and $(X, Y)=\left(x_{i}, k^{\left(n_{i}-1\right) / 2}\right)(i=1, \ldots, n)$ belong to the same class 
$T$ of (2). By Lemma 2, there exists a fixed positive integer solution $\left(X_{0}, Y_{0}\right)$ of (2) which satisfies

$$
\begin{aligned}
x_{i}+k^{\left(n_{i}-1\right) / 2} \sqrt{k}=\left(X_{0}+\delta_{i} Y_{0} \sqrt{k}\right)\left(u_{i}+v_{i} \sqrt{k}\right), & \\
& \delta_{i} \in\{-1,1\}, i=1, \ldots, 5,
\end{aligned}
$$

where $\left(u_{i}, v_{i}\right)(i=1, \ldots, 5)$ are solutions of $(3)$. We find from (18) that

$$
\begin{aligned}
x_{j+1}+\delta_{j+1} k^{\left(n_{j+1}-1\right) / 2} \sqrt{k} & \\
& =\left(x_{j}+\delta_{j} k^{\left(n_{j}-1\right) / 2} \sqrt{k}\right)\left(u_{j}^{\prime}+v_{j}^{\prime} \sqrt{k}\right), \quad j=1, \ldots, 4,
\end{aligned}
$$

where $\left(u_{j}^{\prime}, v_{j}^{\prime}\right)(j=1, \ldots, 4)$ are also solutions of $(3)$. Since $x_{1}<\ldots<x_{5}$, we see from (19) that

$$
\begin{aligned}
x_{j+1}+k^{\left(n_{j+1}-1\right) / 2} \sqrt{k} \\
= \begin{cases}\left(x_{j}+k^{\left(n_{j}-1\right) / 2} \sqrt{k}\right)\left(u_{j}^{\prime \prime}+v_{j}^{\prime \prime} \sqrt{k}\right) & \text { if } \delta_{j}=\delta_{j+1}, \\
\left(x_{j}-k^{\left(n_{j}-1\right) / 2} \sqrt{k}\right)\left(u_{j}^{\prime \prime}+v_{j}^{\prime \prime} \sqrt{k}\right) & \text { if } \delta_{j} \neq \delta_{j+1},\end{cases}
\end{aligned}
$$

$j=1, \ldots, 4$, where $\left(u_{j}^{\prime \prime}, v_{j}^{\prime \prime}\right)$ are positive integer solutions of (3). Notice that $x_{j+1}>x_{j}$ and

$$
\begin{aligned}
\frac{x_{j+1}}{x_{j}} & >\frac{x_{j+1}+k^{\left(n_{j+1}-1\right) / 2} \sqrt{k}}{x_{j}+k^{\left(n_{j}-1\right) / 2} \sqrt{k}} \\
& >\frac{x_{j+1}+k^{\left(n_{j+1}-1\right) / 2} \sqrt{k}}{x_{j}-k^{\left(n_{j}-1\right) / 2} \sqrt{k}}>0, \quad j=1, \ldots, 4 .
\end{aligned}
$$

From (20) and (21), we obtain

$$
\frac{x_{j+1}}{x_{j}}>u_{j}^{\prime \prime}+v_{j}^{\prime \prime} \sqrt{k}, \quad j=1, \ldots, 4 .
$$

On the other hand, by (20), we get

$$
k^{\left(n_{j+1}-1\right) / 2}=x_{j} v_{j}^{\prime \prime} \pm k^{\left(n_{j}-1\right) / 2} u_{j}^{\prime \prime}, \quad j=1, \ldots, 4 .
$$

Since $\operatorname{gcd}(D, k)=\operatorname{gcd}\left(x_{j}, k\right)=1(j=1, \ldots, 4)$, we see from $(23)$ that

$$
k^{\left(n_{j}-1\right) / 2} \mid v_{j}^{\prime \prime}, \quad j=1, \ldots, 4,
$$

and $v_{j}^{\prime \prime} / k^{\left(n_{j}-1\right) / 2}$ is a positive integer satisfying

$$
k^{\left(n_{j+1}-n_{j}\right) / 2}=x_{j} \frac{v_{j}^{\prime \prime}}{k^{\left(n_{j}-1\right) / 2}} \pm u_{j}^{\prime \prime}, \quad j=1, \ldots, 4 .
$$

Since $\operatorname{gcd}\left(u_{j}^{\prime \prime}, k\right)=1(j=1, \ldots, 4)$, from $(25)$ we get

$$
\operatorname{gcd}\left(k, v_{j}^{\prime \prime} / k^{\left(n_{j}-1\right) / 2}\right)=1, \quad j=1, \ldots, 4 .
$$

For $j=1, \ldots, 4$, let $\left(U_{j}, V_{j}\right)$ be the least positive integer solution of (3) such that $k^{\left(n_{j}-1\right) / 2} \mid V_{j}$ and $\operatorname{gcd}\left(k, V_{j} / k^{\left(n_{j}-1\right) / 2}\right)=1$. By Lemma 4 , we deduce 
from (22), (24) and (26) that

$$
\begin{aligned}
\frac{x_{j+2}}{x_{j+1}} & >u_{j+1}^{\prime \prime}+v_{j+1}^{\prime \prime} \sqrt{k} \\
& \geq U_{j+1}+V_{j+1} \sqrt{k}=\left(U_{j}+V_{j} \sqrt{k}\right)^{k^{\left(n_{j+1}-n_{j}\right) / 2}}, \quad j=1,2,3 .
\end{aligned}
$$

By Lemma 1, we have $k^{n_{2}}>\sqrt{D}$. Further, since $k^{\left(n_{2}-1\right) / 2} \mid V_{2}$, we infer from (27) that

$$
x_{3}^{2}>x_{2}^{2}\left(U_{2}+V_{2} \sqrt{k}\right)^{2}>4 x_{2}^{2} k^{n_{2}}>4 x_{2}^{2} \sqrt{D} .
$$

This implies that

$$
k^{n_{3}}=x_{3}^{2}-D>4 x_{2}^{2} \sqrt{D}-D=4\left(D+k^{n_{2}}\right) \sqrt{D}-D>4 D^{3 / 2}+3 D .
$$

Since $k \geq 6$, by the same argument, we can prove that

$$
\begin{aligned}
k^{n_{4}} & =x_{4}^{2}-D>x_{3}^{2}\left(u_{3}^{\prime \prime}+v_{3}^{\prime \prime} \sqrt{k}\right)^{2}-D \geq x_{3}^{2}\left(U_{3}+V_{3} \sqrt{k}\right)^{2}-D \\
& =x_{3}^{2}\left(U_{2}+V_{2} \sqrt{k}\right)^{2 k^{\left(n_{3}-n_{2}\right) / 2}}-D>x_{3}^{2}\left(4 k^{n_{2}}\right)^{k^{\left(n_{3}-n_{2}\right) / 2}}-D \\
& >4 D^{3 / 2}\left(4 D^{1 / 2}\right)^{k}-D>4^{7} D^{9 / 2}-D>4^{3} D^{4},
\end{aligned}
$$

and

$$
\begin{aligned}
k^{n_{5}} & =x_{5}^{2}-D>x_{4}^{2}\left(U_{4}+V_{4} \sqrt{k}\right)^{2}-D \\
& =x_{4}^{2}\left(U_{3}+V_{3} \sqrt{k}\right)^{2 k^{\left(n_{4}-n_{3}\right) / 2}}-D>k^{n_{4}+n_{3} k^{\left(n_{4}-n_{3}\right) / 2}} .
\end{aligned}
$$

We see from $(29)$ that $\left(x_{4}, n_{4}\right)$ is a solution of (1) with $k^{n_{4}}>4^{3} D^{4}$. Moreover, if $D \geq 7$, then we have $k^{n_{4}}>10^{5}$. Since $k$ is not a prime power, $k$ has at least two distinct prime factors $p$ with $(D / p)=1$, where $(D / p)$ is Legendre's symbol. So we have $k \geq 7 \cdot 17,11 \cdot 13$ and $11 \cdot 19$ for $D=2,3$ and 5 respectively. Since $n_{4} \geq 7$, this implies that $k^{n_{4}}>\max \left(10^{5}, 4^{3} D^{4}\right)$. Therefore, by Lemma 6 , we get

$$
39 n_{4}>n_{5} .
$$

The combination of (30) and (31) yields

$$
38 n_{4}>n_{3} k^{\left(n_{4}-n_{3}\right) / 2} .
$$

Since $n_{3} \geq 5$, if $n_{3} \leq n_{4} / 4.6$ then $n_{4} \geq 4.6 n_{3} \geq 23$ and

$$
38 n_{4}>n_{3} k^{9 n_{4} / 23} \geq 5 \cdot 6^{9 n_{4} / 23},
$$

by (32). This is impossible for $n_{4} \geq 23$. If $n_{3}>n_{4} / 4.6$, then from (22) and (32) we get

$$
\begin{aligned}
174.8 n_{4} & >n_{4} k^{\left(n_{4}-n_{3}\right) / 2}=n_{4}\left(\frac{x_{4}^{2}-D}{x_{3}^{2}-D}\right)^{1 / 2}>n_{4} \frac{x_{4}}{x_{3}}>n_{4}\left(U_{3}+V_{3} \sqrt{k}\right) \\
& >2 n_{4} k^{n_{3} / 2}>2 \cdot 6^{5 / 2} n_{4}>176.3 n_{4},
\end{aligned}
$$


a contradiction. Thus, the equation (1) has at most four solutions $\left(x_{i}, n_{i}\right)(i=1, \ldots, 4)$ such that $n_{1}<\ldots<n_{4}, k^{n_{1}}<\sqrt{D}, 2 \nmid n_{i}(i=$ $1, \ldots, 4)$ and $(X, Y)=\left(x_{i}, k^{\left(n_{i}-1\right) / 2}\right)(i=1, \ldots, 4)$ belong to the same class of (2). By the same argument, we can prove that (1) has at most three solutions $\left(x_{i}, n_{i}\right)(i=1, \ldots, 3)$ such that $n_{1}<\ldots<n_{3}, k^{n_{1}}>\sqrt{D}, 2 \nmid n_{i}(i=$ $1, \ldots, 3)$ and $(X, Y)=\left(x_{i}, k^{\left(n_{i}-1\right) / 2}\right)(i=1, \ldots, 3)$ belong to the same class of (2). Further, by Lemma $1,(1)$ has at most one solution $(x, n)$ that satisfies $k^{n}<\sqrt{D}$. This implies that if $D>0$, then (1) has at most $3 \cdot 2^{\omega(D)-1}+1$ solutions $(x, n)$ with $2 \nmid n$. Recall that $(1)$ has at most $2^{\omega(D)-1}$ solutions $(x, n)$ with $2 \mid n$. So we have $N(D, k) \leq 2^{\omega(D)+1}+1$ for $D>0$.

We next consider the case $D<0$. By Lemma 3 , if $-5 \leq D \leq-1$, then $N(D, k) \leq 3$. We may therefore assume that $|D| \geq 6$. Notice that (1) has no solution $(x, n)$ satisfying $k^{n}<|D|$. Therefore, by much the same argument as in the proof of the case $D>0$, we can prove that (1) has at most three solutions $(x, n)$ such that $2 \nmid n$ and $(X, Y)=\left(x, k^{(n-1) / 2}\right)$ belongs to the same class of $(2)$. So we have $N(D, k) \leq 2^{\omega(D)+1}$ for $D<0$. The proof is complete.

\section{References}

[1] R. Apéry, Sur une équation diophantienne, C. R. Acad. Sci. Paris Sér. A 251 (1960), 1263-1264.

[2] - Sur une équation diophantienne, ibid., 1451-1452.

[3] F. Beukers, On the generalized Ramanujan-Nagell equation I, Acta Arith. 38 (1981), 389-410.

[4] -, On the generalized Ramanujan-Nagell equation II, ibid. 39 (1981), 113-123.

[5] E. Brown, The diophantine equation of the form $x^{2}+D=y^{n}$, J. Reine Angew. Math. 274/275 (1975), 385-389.

[6] X.-G. Chen and M.-H. Le, On the number of solutions of the generalized Ramanujan-Nagell equation $x^{2}-D=k^{n}$, Publ. Math. Debrecen, to appear.

[7] L.-K. Hua, Introduction to Number Theory, Springer, Berlin, 1982.

[8] M.-H. Le, On the generalized Ramanujan-Nagell equation $x^{2}-D=p^{n}$, Acta Arith. 58 (1991), 289-298.

[9] - On the number of solutions of the generalized Ramanujan-Nagell equation $x^{2}-$ $D=2^{n+2}$, ibid. 60 (1991), 149-167.

[10] - Sur le nombre de solutions de l'équation diophantienne $x^{2}+D=p^{n}$, C. R. Acad. Sci. Paris Sér. I Math. 317 (1993), 135-138.

[11] - Some exponential diophantine equations $I$ : The equation $D_{1} x^{2}-D_{2} y^{2}=\lambda k^{z}$, J. Number Theory 55 (1995), 209-221.

[12] V. A. Lebesgue, Sur l'impossibilité, en nombres entiers, de l'équation $x^{m}=y^{2}+1$, Nouv. Ann. Math. (1) 9 (1850), 178-181.

[13] T. Nagell, Contributions to the theory of a category of diophantine equations of the second degree with two unknowns, Nova Acta R. Soc. Sc. Uppsal. (4) 16 (1954), No. 2. 
[14] N. Tzanakis and J. Wolfskill, On the diophantine equation $y^{2}=4 q^{n}+4 q+1$, J. Number Theory 23 (1986), 219-237.

[15] T.-J. Xu and M.-H. Le, On the diophantine equation $D_{1} x^{2}+D_{2}=k^{n}$, Publ. Math. Debrecen 47 (1995), 293-297.

Department of Mathematics Zhanjiang Teachers College

524048 Zhanjiang, Guangdong

P.R. China

Received on 29.8.1995

and in revised form on 18.3 .1996 